\title{
Desapariciones y desenlaces suicidas. Un análisis del método, entorno y desplazamiento
}

Going missing and suicide outcomes. An analysis of method, location and distance travelled.

\author{
Laura Larrañaga Casquero ${ }^{1}$ \\ María Alfaro Coronado ${ }^{1}$ \\ Néstor García Barceló ${ }^{2}$ \\ José Luis González Álvarez ${ }^{3}$
}

(1) Universidad Complutense de Madrid, Madrid, España.

(2) Instituto de Ciencias Forenses y de la Seguridad, Universidad Autónoma de Madrid, Madrid, España. (3) Secretaria de Estado de Seguridad, Ministerio del Interior, Madrid, España

Email de correspondencia: nestor.garcia@icfs-uam.es

\begin{abstract}
Resumen
Las desapariciones son un fenómeno global que generan una gran alarma social y suponen una repercusión psicológica grave para los implicados. La mayoría de personas desaparecidas son localizadas en menos de 24 horas en buen estado de salud. No obstante, un pequeño porcentaje se asocia con un desenlace fatal, siendo el suicidio la principal causa de muerte. El trabajo actual se centra en el estudio de las desapariciones con desenlace suicida en función del sexo y la edad de la persona desaparecida. Para ello, se ha realizado un análisis del método empleado, entorno utilizado y distancia recorrida por la persona, intentando establecer si existe una asociación entre las variables. Los resultados muestran que los hombres tienden a utilizar el aborcamiento y los entornos boscosos, desplazándose mayores distancias, mientras que las mujeres recorren una menor distancia destacando la sobredosis como método predilecto. No se han encontrado resultados concluyentes en cuanto a método y entorno según la edad del individuo pero si se ha observado que la distancia recorrida es menor cuanto mayor edad tiene el desaparecido. Este estudio supone un aumento del conocimiento empirico en torno a las desapariciones de etiología suicida, aportando información que pueda ser utilizada en las estrategias preventivas, asi como de actuación policial.
\end{abstract}

Palabras Clave: Desaparecidos, suicidio, método, entorno, distancia recorrida.

\begin{abstract}
Going missing is considered a global phenomenon that generates a great concern in society and has a great psychological impact on the people involved. In spite of the fact that most people that go missing are located within 24 hours in good health conditions, there is a percentage of cases associated to a fatal outcome, with suicide identified as the leading cause of death. The current research focuses on the study of missing people with a suicide outcome according to the missing person's sex and age. By using a statistical and descriptive analysis of the chosen methods, locations and distance travelled, an association between these variables has been tried to establish. The results found show that men tend to use hanging and woodlands, therefore travelling a longer distance, while women are more likely to overdose and travel a shorter distance. There were no consistent results for method and location according to age, although it was possible to observe that the travelled distance was shorter the older the missing person was. This study shows an increase of the empirical knowledge on the phenomenon of going missing with a suicide outcome, providing information that may be used in preventive strategies, as well as in police response.
\end{abstract}

Keywords: Missing people, suicide, method, location, distance travelled. 


\section{INTRODUCCIÓN}

Las desapariciones son una problemática universal que, en determinados casos, genera una gran alarma social y que requiere la preocupación y actuación de las Fuerzas y Cuerpos de Seguridad del Estado (FCSE) ya que cualquier persona puede desaparecer y resultar dañada durante ese periodo de ausencia. Además, también se debe tener en cuenta la repercusión psicológica grave de las personas implicadas así como el despliegue de recursos policiales que se lleva a cabo para localizar a la persona de la forma más rápida posible intentando evitar un desenlace fatal (García- Barceló, Tourís \& González, 2019).

A nivel internacional, se entiende que una persona desaparecida es aquella "cuya localización no puede ser establecida y cuy as circunstancias se encuentran en contradicción con el comportamiento habitual de la misma, o que el contexto sugiere que la persona puede ser víctima de un delito o encontrarse en riesgo de dañarse a si mismo o a otros", mientras que, "ausente", se entiende como "la persona que no se encuentra en el lugar donde se le espera sin que el contexto sugiera que la persona puede ser victima de un delito o encontrarse en riesgo de dañarse a si mismo o a otros" (ACPO, 2013).

Las desapariciones son un fenómeno global. En 2017 se registraron en EEUU un total de 651226 denuncias por desaparición (National Crime Information Center, FBI, 2017); la National Crime Agency UK Missing persons Bureau registra anualmente 300000 casos en Reino Unido (Taylor, Woolnough \& Dickens, 2018) mientras que Australia cuantifica 35000 denuncias al año (Stevenson y Thomas, 2018). En España, se registraron un total de 29740 denuncias por desaparición en 2018. A 31 de diciembre de 2018, se encontraban un total de 12330 denuncias activas desde 2010 (Ministerio del Interior, 2019).

En nuestro país, la preocupación social sobre el fenómeno de las personas desaparecidas impulsó la necesidad de una respuesta institucional especializada. Entre las iniciativas desarrolladas a tal efecto destaca la creación del Centro Nacional de Desaparecidos (CNDES), siendo este el órgano responsable de la gestión centralizada y coordinación del sistema empleado por las Fuerzas y Cuerpos de Seguridad (Ministerio del Interior, 2019). Además, se han dictado una serie de instrucciones por la Secretaría de Estado de Seguridad: Inst. 1/2009 sobre la "actuación policial ante la desaparición de menores de edad y otras desapariciones de alto riesgo";
Inst.14/2014, que regula el "procedimiento para la activación y funcionamiento del sistema de alerta temprana por desaparición de menores"; Inst. 1/2017 que actualiza el "protocolo de actuación policial con menores", y la Inst. 3/2019 que publica el "nuevo protocolo de actuación policial ante la desaparición de personas".

La demanda por una respuesta policial rápida y efectiva viene avalada por el hecho de que, a pesar de que la mayoría de personas desaparecidas son localizadas dentro de las primeras 24 horas (Fyfe, Stevenson y Woolnough, 2015), en buen estado de salud física y mental (Foy 2006), entre el 1-3\% de desapariciones se asocian con un desenlace fatal siendo el suicidio la principal causa de muerte en este fenómeno (Foy, 2016; Newiss, 2011).

Las FCSE tienen la obligación de priorizar los casos más graves, es decir, aquellos que tengan riesgo de terminar con un daño fatal. En los casos de las personas desaparecidas con intención de suicidarse, uno de los objetivos de las FCSE es evitar este hecho debiendo iniciar la búsqueda de la persona de una manera activa (Domenech, 2018). En consecuencia, el estudio del método utilizado, el entorno elegido y la distancia recorrida por las personas que desaparecen con la intención de suicidarse, puede revelar información fundamental que ayude a establecer un área de búsqueda abreviando el tiempo de respuesta de las FCSE, logrando llegar a la persona desaparecida antes de que se dañe a sí misma y previniendo dicho acto.

\section{Antecedentes teóricos}

La investigación científica en torno a las desapariciones ha sido relativamente escasa, y se ha tendido a priorizar el estudio de las desapariciones que afectan a los menores, por su especial vulnerabilidad, dejando de lado aquellas que atañen a las personas adultas (Bonny, Almond y Woolnough, 2016; Biehal, Mitchell y Wade, 2003; Shalev, Schaefer y Morgan, 2009). En los últimos años se ha producido un progresivo interés en el estudio de esta materia, lo que ha quedado reflejado con el incremento del número de publicaciones científicas (Taylor et al., 2018).

En general, la literatura académica coincide en que la mayoría de personas que desaparecen son adolescentes, entre los 14 a 18 años aproximadamente, y que suelen estar vinculados a desapariciones voluntarias como fugas (Tarling \& Barrows, 2004; Foy, 2016; Newiss, 2006; Fyfe et al., 2015). Entre los adultos, el grupo de edad que más tiende a desaparecer son los individuos de entre 24 y 30 
años, decreciendo gradualmente el número de casos conforme se incrementa la edad (Biehal et al., 2003). Analizando el sexo de la persona desaparecida, aunque es un fenómeno que por lo general afecta más a hombres, no se suelen encontrar diferencias significativas (Foy, 2016).

Siguiendo esta línea, uno de los aspectos en los que ha puesto el foco de interés la investigación científica reside en el estudio de aquellas desapariciones en las que la persona es localizada sin vida: causa natural, accidental, suicida y homicida. El estudio de este tipo de desenlace puede generar diversas implicaciones en el ámbito de la prevención e intervención policial (García-Barceló et al., 2019). Algunos estudios señalan que los hombres tienen un mayor riesgo de ser localizados sin vida que las mujeres, así como que la probabilidad de que una persona desaparecida sea localizada sin vida aumenta con la edad (Newiss, 2006).

En cuanto a la investigación en torno al suicidio, en los últimos años se ha descrito como un continuo de diferente naturaleza y gravedad, que va desde la ideación y los intentos, hasta el suicidio consumado (Tang, Wu \& Miao, 2013). Considerando el sexo, el suicidio consumado prevalece en los varones, aunque lo intentan más las mujeres (Abellán, 2005; Greene, Schafer \& Morgan, 2009; Perkins, Roberts \& Feeney, 2011). En lo que al estudio de la edad respecta, las tasas de suicidio consumado aumentan con la misma (De Leo, Conforti \& Carollo, 1997; Skogman, Alsén \& Öjehagen, 2004; Tadros \& Salib, 2000), aunque no hay que olvidar que entre la población juvenil es un fenómeno creciente y que es la segunda causa de muerte en jóvenes de entre 15 y 29 años (OMS, 2014).

En la investigación de las desapariciones y el suicidio se ha puesto el foco de atención en tres aspectos: el entorno y el método utilizado, así como el desplazamiento de las personas desaparecidas para cometer el suicidio (Gibb \& Woolnough, 2007; Woolnough, Magar \& Graham, 2019).

El método utilizado se encuentra vinculado a la accesibilidad, relacionado tanto con el entorno espacial en el que se mueve el individuo como con los elementos necesarios de los que dispone (Gibb y Woolnough, 2007). Woolnough et al., (2019) señalan que los métodos más prevalentes en el ámbito de las desapariciones cuyo desenlace es suicida son ahorcamiento (50\%) y ahogamiento (28\%).

Según el sexo del individuo, las investigaciones han podido establecer que los hombres tienden a utilizar métodos más violentos que aseguran el éxito letal, prevaleciendo el ahorcamiento, mientras que en mujeres es más probable el envenenamiento o el ahogamiento (Bergen et al. 2012; Callanan \& Davis, 2011; Gibb \& Woolnough, 2007; Hawton \& Heeringen, 2009).

En cuanto a la edad, se conoce que los individuos más longevos tienden a utilizar métodos que requieren de un menor esfuerzo físico, por circunstancias como la falta de energía, la depresión o enfermedades físicas (Tadros \& Salib, 2000).

Por otro lado, el entorno en el que la persona decide suicidarse, entendido como el lugar en el que el individuo se suicida y no necesariamente siendo el elemento causante de su muerte (domicilio, vehículo, entorno acuático, etcétera), es también un gran proveedor de información, ya que en muchas ocasiones el entorno va a limitar la accesibilidad al método. Las razones por las que unos y otros escogen según qué zona para suicidarse pueden ser variadas aunque, por lo general, parecen deberse a dos principales causas: que el espacio elegido contemple aspectos de carácter práctico para cometer el acto, o que tenga una significación personal (Gibb \& Woolnough, 2007). Woolnough et al., (2019) señalan que aquellas personas desaparecidas que cometieron el suicidio escogieron como entorno el domicilio en el 65\% de las ocasiones, mientras que lo hicieron en entornos naturales (acuáticos y boscosos) en un 23.8\% de las veces.

Respecto al sexo, la Agencia Nacional del Crimen del Reino Unido (2016) refleja que la mayoría de hombres escogen los bosques y entornos acuáticos para suicidarse (un 28\% y $26 \%$ respectivamente), mientras que las mujeres tienden a suicidarse en áreas locales exteriores (26\%), bosques $(21 \%)$ y vehículos $(21 \%)$, principalmente.

La distancia recorrida por el sujeto para llegar al entorno adecuado también tiene una gran relevancia. Sveticic, San Too y De Leo (2012) encontraron que los suicidas desaparecidos, tendían a ubicarse en lugares naturales al aire libre, en contraste con los suicidios no denunciados, que tienden a ocurrir en la propia casa. Asimismo, Woolnough et al., (2019), descubrieron que las personas que cometieron el suicidio y que habían desaparecido anteriormente recorrieron una mayor distancia media $(32 \mathrm{~km})$ que aquellas personas sobre las que no se había interpuesto denuncia por desaparición (13 $\mathrm{km})$.

Gibb y Woolnough (2007) establecieron una relación entre el sexo y la distancia que recorrieron los suicidas cuya 
Larrañaga, L., Alfaro, M., García, N. y González, J. L.. (2019). Desapariciones y desenlaces suicidas. Un análisis del método, entorno y desplazamiento. Behavior \& Law Journal, 5(1), 9-17.

desaparición había sido denunciada, destacando que las mujeres se desplazaron más que los hombres. El 90\% de los hombres no se desplazó más de $2.4 \mathrm{~km}$ a pie mientras que las mujeres ampliaron la distancia máxima hasta 6.9 $\mathrm{km}$. Por otro lado, el 40\% de las mujeres se desplazó más de $20 \mathrm{~km}$ cuando usaron un vehículo a motor mientras que solo el 20\% de los hombres superó dicha distancia (Gibb \& Woolnough, 2007). En contraposición, la National Crime Agency (2016) publicó un estudio que señalaba que los hombres recorrían mayores distancias para suicidarse que las mujeres. Ninguna de las mujeres a pie recorrió más de $2.2 \mathrm{~km}$ mientras que aproximadamente el 50\% de los hombres recorrieron esta distancia. En lo referido a cuando usan transporte, los resultados fueron parecidos. Las mujeres no alcanzaron los $35 \mathrm{~km}$ de distancia y el $80 \%$ de los hombres se mantuvieron dentro de los $30 \mathrm{~km}$.

En cuanto a la edad, los estudios señalan que la distancia recorrida por los mayores de edad también es mayor que aquella recorrida por menores de 9 años (National Crime Agency, 2016). Sin embargo, se desconoce si existe una relación entre el desplazamiento y la edad cuando hablamos de personas desaparecidas mayores de 18 años puesto que la literatura no ha profundizado más allá de la diferencia entre mayores y menores de edad.

\section{OBJETIVOS}

La escasez en el estudio del fenómeno de las desapariciones genera la necesidad de dedicar más esfuerzos a su investigación, especialmente en nuestro país. El objetivo de este trabajo es aportar conocimiento empírico al fenómeno que pueda ser utilizado en el establecimiento de estrategias preventivas, así como de actuación policial, contribuyendo a la prevención y reducción del comportamiento suicida en el ámbito de las desapariciones.

Para ello se pretende llevar a cabo un análisis del método, entorno y desplazamiento de casos de personas desaparecidas con desenlace suicida en España, en relación con las variables demográficas sexo y edad.

\section{HIPÓTESIS}

Tras la lectura de la literatura científica existente se contemplan las siguientes hipótesis:
1. El ahorcamiento será el método prevalente entre los hombres y la sobredosis entre las mujeres.

2. El ahorcamiento se verá incrementado con la edad.

b) Entorno elegido:

3. Los hombres tenderán a acudir a entornos exteriores y las mujeres a interiores.

4. Se tenderá a usar entornos exteriores a menor edad y a emplear lugares interiores a mayor edad.

c) Desplazamiento para llevar a cabo el suicidio:

5. Los hombres se desplazarán una mayor distancia que las mujeres.

6. Cuanta mayor edad tenga la persona desaparecida, menor será la distancia recorrida.

\section{METODOLOGÍA}

\section{Muestra}

La muestra para este estudio se compone de un total de 43 casos de desapariciones que terminaron con el suicidio de la persona desaparecida y que fueron denunciados previamente entre 2011 y 2019 ante la Guardia Civil y la Policía Nacional.

Del total, 13 son mujeres (30.23\%) y 30 hombres (69.77\%), 42 son nacionales $(97.67 \%)$ y una persona de procedencia extranjera (2.33\%; ver la Tabla 1$)$. La muestra está compuesta en su totalidad por personas mayores de edad, situándose la media de edad en 51.26 años (DT $=12.855, \operatorname{mdn}=50)$ y presentando un rango de 51 años, situándose el mínimo en 30 años y el máximo en 81 (ver la Tabla 2). Teniendo en cuenta el tamaño de la muestra, se realizó la prueba de normalidad Shapiro Wilk mostrando que la edad seguía una distribución normal $(\mathrm{p}>0.05)$.

Tabla 1. Descripción de la muestra

\begin{tabular}{lccc}
\hline \hline \multicolumn{1}{c}{ Variable } & & $\mathrm{n}$ & $\%$ \\
\hline \hline \multirow{2}{*}{ Sexo $(\mathrm{n}=43)$} & Mujer $(\mathrm{M})$ & 13 & 30.23 \\
\hline \multirow{2}{*}{ Edad $(\mathrm{n}=43)$} & Hombre $(\mathrm{H})$ & 30 & 69.77 \\
\hline \multirow{2}{*}{ Nacionalidad $(\mathrm{n}=43)$} & Mayor de edad & 43 & 100 \\
\cline { 2 - 4 } & Menor de edad & & \\
\cline { 2 - 4 } & Nacional & 42 & 97.67 \\
\hline \hline
\end{tabular}

a) Método utilizado: 
Larrañaga, L., Alfaro, M., García, N. y González, J. L.. (2019). Desapariciones y desenlaces suicidas. Un análisis del método, entorno y desplazamiento. Behavior \& Law Journal, 5(1), 9-17.

Tabla 2. Descripción de la edad

\begin{tabular}{lc}
\hline \hline & Edad $(\mathrm{n}=43)$ \\
\hline \hline Media & 51.26 \\
\hline Mediana & 50 \\
\hline Desviación típica & 12.855 \\
\hline Rango & 51 \\
\hline Mínimo & 30 \\
\hline Máximo & 81 \\
\hline \hline
\end{tabular}

Procedimiento y análisis de datos

En primer lugar se realizó una revisión de la literatura científica existente.

Posteriormente se procedió a la solicitud, recepción, lectura y depurado de 43 atestados sobre denuncias por desaparición realizadas en territorio español tanto de Guardia Civil (6) como de Policía Nacional (37) cuyo desenlace había sido fatal y se presuponía el suicidio como etiología de la muerte.

Paralelamente se creó una base de datos con el programa SPSS donde se incluyeron las variables de estudio: método, entorno y distancia recorrida. La clasificación del método y del entorno se realizó teniendo en cuenta las propuestas de la literatura académica. Para la recogida del desplazamiento se grabaron los lugares de desaparición y los de localización del cadáver a través de la geolocalización de los mismos con la herramienta Google Maps. Posteriormente se calculó la distancia euclídea entre éstos.

Finalmente se procedió al análisis estadístico descriptivo e inferencial de los datos, empleando diferentes estadísticos: el test de independencia $\chi^{2}$, el test de Fisher, la U de Mann-Whitney, la Correlación de Spearman y la Correlación de Kendall.

\section{RESULTADOS}

De los 43 casos originales, solo se pudo establecer el método utilizado en 37. Los resultados muestran cómo los métodos prevalentes que los desaparecidos utilizan para llevar a cabo el acto de suicidarse son el ahorcamiento (40.5\%), la sobredosis (18.9\%) y el ahogamiento (16.2\%). Concretamente, el ahorcamiento se encuentra más asociado a los hombres (50\%), así como la precipitación $(16.7 \%)$. Las mujeres tienden a escoger la sobredosis como método de suicidio (38.5\%), seguido del ahorcamiento y el ahogamiento, ambos en la misma proporción (23.1\%; ver tabla 3).
Tabla 3. Distribución del método de suicidio entre hombres $(\mathrm{H})$ y mujeres (M).

\begin{tabular}{cccc}
\hline \hline Método $(\mathrm{n}=37)$ & $\mathrm{M}$ & $\mathrm{H}$ & Total \\
\hline \hline Ahorcamiento & $3(23.1 \%)$ & $12(50 \%)$ & $15(40.5 \%)$ \\
\hline Sobredosis & $5(38.5 \%)$ & $2(8.3 \%)$ & $7(18.9 \%)$ \\
\hline Ahogamiento & $3(23.1 \%)$ & $3(12.5 \%)$ & $6(16.2 \%)$ \\
\hline Precipitación & & $4(16.7 \%)$ & $4(10.8 \%)$ \\
\hline Arroyamiento & $2(5.4 \% \%)$ & $1(2.7 \%)$ & $3(8.1 \%)$ \\
\hline Arma de fuego & & $2(8.3 \%)$ & $2(5.4 \%)$ \\
\hline Total & $13(100 \%)$ & $24(100 \%)$ & $37(100 \%)$ \\
\hline \hline
\end{tabular}

La determinación del entorno ha sido posible para un total de 38 casos. El análisis descriptivo de los datos indica cómo el domicilio (18.4\%) y el entorno acuático (18.4\%) son los entornos prevalentes, seguido del entorno boscoso $(15.8 \%)$ y la vía pública (13.2\%), tal y como se presenta en la tabla 4. Entre los hombres el entorno prevalente es el entorno boscoso (20\%), sin ser este un entorno frecuentemente escogido por las mujeres (7.7\%). También la vía pública es utilizada por los hombres (16\%) con mayor frecuencia que las mujeres $(7.7 \%)$.

Tabla 4. Distribución del entorno de suicidio entre hombres y mujeres

\begin{tabular}{lccc}
\hline \hline \multicolumn{1}{c}{ Entornos $(\mathrm{n}=38)$} & $\mathrm{M}$ & $\mathrm{H}$ & Total \\
\hline \hline Domicilios & $3(23.1 \%)$ & $4(16 \%)$ & $7(18.4 \%)$ \\
\hline Entorno acuático & $3(23.1 \%)$ & $4(16 \%)$ & $7(18.4 \%)$ \\
\hline Entorno boscoso & $1(7.7 \%)$ & $5(20 \%)$ & $6(15.8 \%)$ \\
\hline Vía pública & $1(7.7 \%)$ & $4(16 \%)$ & $5(13.2 \%)$ \\
\hline Estación de tren o metro & $2(15.4 \%)$ & $2(8 \%)$ & $410.5 \%)$ \\
\hline Hotel & $2(15.4 \%)$ & & $2(5.3 \%)$ \\
\hline Entorno montañoso & & $2(8 \%)$ & $2(5.3 \%)$ \\
\hline Otros & & $2(8 \%)$ & $2(5.3 \%)$ \\
\hline Vehículo & $1(7.7 \%)$ & $1(4 \%)$ & $2(5.3 \%)$ \\
\hline Entorno laboral & $1(4 \%)$ & $1(2.6 \%)$ \\
\hline Total & $13(100 \%)$ & $25(100 \%)$ & $38(100 \%)$ \\
\hline \hline
\end{tabular}

A partir de una segunda clasificación del entorno empleado se ha podido establecer que los entornos situados en el exterior (63.2\%) son más frecuentemente utilizados que los interiores (36.8\%) (ver tabla 5). Concretamente, los hombres tienden a escoger un entorno situado en el exterior para llevar a cabo el suicidio (72\%) con mayor frecuencia que las mujeres (46.2\%), mientras que estas últimas tienden a escoger entornos en el interior $(53.8 \%)$ con mayor frecuencia que los hombres (28\%).

Tabla 5. Distribución del entorno (interior/exterior) entre hombres y mujeres

\begin{tabular}{lccc}
\hline \hline $\begin{array}{c}\text { Entorno } \\
(\mathrm{n}=38)\end{array}$ & $\mathrm{M}$ & $\mathrm{H}$ & Total \\
\hline \hline Interior & $7(53.8 \%)$ & $7(28 \%)$ & $14(36.8 \%)$ \\
\hline Exterior & $6(46.2 \%)$ & $18(72 \%)$ & $24(63.2 \%)$ \\
\hline Total & $13(100 \%)$ & $25(100 \%)$ & $38(100 \%)$ \\
\hline \hline
\end{tabular}

Tras realizar el test de independencia $\chi^{2}$ y el Test de Fisher no se encontraron resultados estadísticamente significativos entre las variables entorno, método y sexo. 
Larrañaga, L., Alfaro, M., García, N. y González, J. L.. (2019). Desapariciones y desenlaces suicidas. Un análisis del método, entorno y desplazamiento. Behavior Æ Law Journal, 5(1), 9-17.

En cuanto al análisis del desplazamiento, han sido excluidos 4 casos (3 al encontrarse la víctima en el extranjero y 1 al desconocer el lugar exacto de localización del cadáver). Analizados los datos, se encontró que la distancia mínima recorrida fue de 0 metros, y la máxima fue de $180.15 \mathrm{~km}$ (media $=15.07 \mathrm{~km} ; m d n=2.31 \mathrm{~km}$ ). Se realizó la prueba de normalidad Shapiro Wilk mostrando que la distancia siguió una distribución no normal $(\mathrm{p}<0.05)$.

Centrándonos en la distancia recorrida, dependiendo del sexo del desaparecido (ver Figura 1), se observó que el $69.3 \%$ de las mujeres recorrió una distancia menor a $3 \mathrm{~km}$ para llevar a cabo el suicidio. Un $23 \%$ se desplazó entre 4 y $10 \mathrm{~km}$ y el $7.7 \%$ de las mujeres más de $21 \mathrm{~km}$. Sin embargo, en el caso de los hombres, el 53.8\% recorrió una distancia inferior a $3 \mathrm{~km}$, un 7.7\% se desplazó entre 4 y 10 $\mathrm{km}$, un $23 \%$ entre 11 y $20 \mathrm{~km}$, y el $15.5 \%$ de los hombres se desplazó más de $21 \mathrm{~km}$.

\section{Figura 1. Distancia recorrida según el sexo}

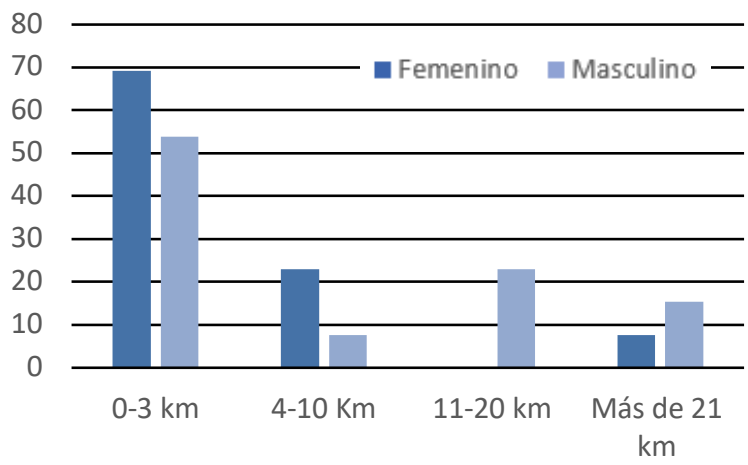

Atendiendo a la mediana, los hombres se desplazaron más $(\mathrm{mdn}=2.8 \mathrm{~km})$ que las mujeres $(\mathrm{mdn}=1.8 \mathrm{~km})$. No obstante, se realizó la prueba no paramétrica U de MannWhitney para comparar estadísticamente las variables sexo y distancia, sin encontrar resultados estadísticamente significativos $(\mathrm{p}>0.05)$.

Además, se estudió la variable edad recodificada en rangos según los establecidos en la literatura científica $(<30,30-49,50-69,>70)$. En la muestra estudiada no existían menores de edad, lo que se debe en cierta medida a la baja prevalencia de los suicidios para este grupo de edad. Se pudo establecer que el ahorcamiento es el método más frecuentemente utilizado para cada rango de edad (40.5\%; ver tabla 6). En el rango de 30 a 49 años destacan, además del ahorcamiento (35.3\%), la sobredosis $(23.5 \%)$ y el ahogamiento (17.6\%). Entre 50 y 69 años, destaca el ahorcamiento como método prevalente $(37.5 \%)$, la sobredosis (18.8\%) y la precipitación (18.8\%). Finalmente, el grupo de individuos de más de 70 años empleó únicamente el ahorcamiento (75\%) y el ahogamiento $(25 \%)$.

Tabla 6. Distribución del método según la edad

\begin{tabular}{ccccc}
\hline \hline $\begin{array}{c}\text { Método } \\
(\mathrm{n}=37)\end{array}$ & \multicolumn{3}{c}{ Rangos de edad } & Total \\
\hline \hline \multirow{2}{*}{ Ahorcamiento } & $30-49$ & $50-69$ & $70+$ & \\
& $(35.3 \%)$ & $(37.5 \%)$ & $(75 \%)$ & $(40.5 \%)$ \\
\hline \multirow{2}{*}{ Sobredosis } & 4 & 3 & & 7 \\
& $(23.5 \%)$ & $(18.8 \%)$ & & $(18.9 \%)$ \\
\hline \multirow{2}{*}{ Ahogamiento } & 3 & 2 & 1 & 6 \\
& $(17.6 \%)$ & $(12.5 \%)$ & $(25 \%)$ & $(16.2 \%)$ \\
\hline \multirow{2}{*}{ Precipitación } & 1 & 3 & & 4 \\
& $(5.9 \%)$ & $(18.8 \%)$ & & $(10.8 \%)$ \\
\hline \multirow{2}{*}{ Arroyamiento } & 2 & 1 & & 3 \\
& $(11.8 \%)$ & $(6.3 \%)$ & & $(8.1 \%)$ \\
\hline \multirow{2}{*}{ Arma de fuego } & 1 & 1 & & 2 \\
& $(5.9 \%)$ & $(6.3 \%)$ & & $(5.4 \%)$ \\
\hline \multirow{2}{*}{ Total } & 17 & 16 & 4 & 37 \\
& $(100 \%)$ & $(100 \%)$ & $(100 \%)$ & $(100 \%)$ \\
\hline \hline
\end{tabular}

En cuanto al análisis del entorno según la edad del individuo, destacan principalmente el entorno acuático $(25 \%)$ y el entorno boscoso $(25 \%)$ para los individuos de 30 a 49 años, y la vía pública (27.8\%) para los individuos de 50 a 69 años (ver tabla 7). Los entornos para el grupo de individuos más avanzados en edad se han distribuido de manera regular entre el domicilio, el entorno acuático, la estación y la categoría "otros".

Tabla 7. Distribución del entorno según la edad

\begin{tabular}{|c|c|c|c|c|}
\hline \multirow[t]{2}{*}{ Entorno $(\mathrm{n}=38)$} & \multicolumn{3}{|c|}{ Rangos de edad } & \multirow[t]{2}{*}{ Total } \\
\hline & $30-49$ & $50-69$ & $70+$ & \\
\hline Domicilios & $\begin{array}{c}3 \\
(18.8 \%)\end{array}$ & $\begin{array}{c}3 \\
(16.7 \%)\end{array}$ & $\begin{array}{c}1 \\
(25 \%)\end{array}$ & $\begin{array}{c}7 \\
(18.4 \%)\end{array}$ \\
\hline Entorno acuático & $\begin{array}{c}4 \\
(25 \%) \\
\end{array}$ & $\begin{array}{c}2 \\
(11.1 \%) \\
\end{array}$ & $\begin{array}{c}1 \\
(25 \%) \\
\end{array}$ & $\begin{array}{c}7 \\
(18.4 \%) \\
\end{array}$ \\
\hline Entorno boscoso & $\begin{array}{c}4 \\
(25 \%)\end{array}$ & $\begin{array}{c}2 \\
(11.1 \%) \\
\end{array}$ & & $\begin{array}{c}6 \\
(15.8 \%)\end{array}$ \\
\hline Vía pública & & $\begin{array}{c}5 \\
(27.8 \%)\end{array}$ & & $\begin{array}{c}5 \\
(13.2)\end{array}$ \\
\hline $\begin{array}{l}\text { Estación de } \\
\text { tren/metro }\end{array}$ & $\begin{array}{c}2 \\
(12.5 \%) \\
\end{array}$ & $\begin{array}{c}1 \\
(5.6 \%) \\
\end{array}$ & $\begin{array}{c}1 \\
(25 \%) \\
\end{array}$ & $\begin{array}{c}4 \\
(10.5 \%) \\
\end{array}$ \\
\hline Entorno montañoso & $\begin{array}{c}1 \\
(6.3 \%) \\
\end{array}$ & $\begin{array}{c}1 \\
(5.6 \%) \\
\end{array}$ & & $\begin{array}{c}2 \\
(5.3 \%) \\
\end{array}$ \\
\hline Hotel & $\begin{array}{c}1 \\
(6.3 \%)\end{array}$ & $\begin{array}{c}1 \\
(5.6 \%)\end{array}$ & & $\begin{array}{c}2 \\
(5.3 \%)\end{array}$ \\
\hline Otros & & $\begin{array}{c}1 \\
(5.6 \%) \\
\end{array}$ & $\begin{array}{c}1 \\
(25 \%) \\
\end{array}$ & $\begin{array}{c}2 \\
(5.3 \%) \\
\end{array}$ \\
\hline Vehículo & $\begin{array}{c}1 \\
(6.3 \%)\end{array}$ & $\begin{array}{c}1 \\
(5.6 \%)\end{array}$ & & $\begin{array}{c}2 \\
(5.3 \%)\end{array}$ \\
\hline Entorno laboral & & $\begin{array}{c}1 \\
(5.6 \%) \\
\end{array}$ & & $\begin{array}{c}1 \\
(2.6 \%) \\
\end{array}$ \\
\hline Total & $\begin{array}{c}16 \\
(100 \%) \\
\end{array}$ & $\begin{array}{c}18 \\
(100 \%)\end{array}$ & $\begin{array}{c}4 \\
(100 \%) \\
\end{array}$ & $\begin{array}{c}38 \\
(100 \%) \\
\end{array}$ \\
\hline
\end{tabular}

En una segunda clasificación del entorno empleado se ha podido establecer que el grupo de edad que acude al exterior con mayor frecuencia es el comprendido por individuos de 50 a 69 años (66.7\%), seguido por el grupo de 30 a 49 años (62.5\%; ver tabla 8). Finalmente, para el 
Larrañaga, L., Alfaro, M., García, N. y González, J. L.. (2019). Desapariciones y desenlaces suicidas. Un análisis del método, entorno y desplazamiento. Behavior \& Law Journal, 5(1), 9-17.

grupo más avanzado en edad, no se han encontrado diferencias, utilizando ambos tipos de entorno con la misma frecuencia $(50 \%)$.

Tabla 8. Distribución del entorno (interior/exterior) según la edad

\begin{tabular}{ccccc}
\hline \hline Entorno $(\mathrm{n}=38)$ & \multicolumn{3}{c}{ Rangos de edad } & Total \\
\hline \hline \multirow{2}{*}{ Interior } & $30-49$ & $50-69$ & $70+$ & \\
& 6 & 6 & 2 & 14 \\
& $(37.5 \%)$ & $(33.3 \%)$ & $(50 \%)$ & $(36.8 \%)$ \\
\hline \multirow{2}{*}{ Exterior } & 10 & 12 & 2 & 24 \\
& $(62.5 \%)$ & $(66.7 \%)$ & $(50 \%)$ & $(63.2 \%)$ \\
\hline \multirow{2}{*}{ Total } & 16 & 18 & 4 & 38 \\
& $(100 \%)$ & $(100 \%)$ & $(100 \%)$ & $(100 \%)$ \\
\hline \hline
\end{tabular}

Tras realizar el test de independencia $\chi^{2}$ y el Test de Fisher no se encontraron resultados estadísticamente significativos entre las variables entorno, método y edad.

Respecto a la distancia recorrida, según la edad del desaparecido (ver Figura 2), se encontró que el 53\% de las personas desaparecidas entre 30 y 49 años no se desplazaron más de $3 \mathrm{~km}$, el 29,4\% entre 4 y $10 \mathrm{~km}$, el $11.7 \%$ entre 11 y $20 \mathrm{~km}$, y el 5.9\% recorrió una distancia mayor a $21 \mathrm{~km}$. Considerando la edad comprendida entre 50 y 69 años, se observó que el 61,1\% se desplazó menos de $3 \mathrm{~km}$, el $22.2 \%$ entre 11 y $20 \mathrm{~km}$ y el $16.7 \%$ viajó más de $21 \mathrm{~km}$. En el caso de las personas mayores de 70 años, el 75\% se desplazó menos de 3 kilómetros y el 25\% más de $21 \mathrm{~km}$.

\section{Figura 2. Distancia según la edad.}

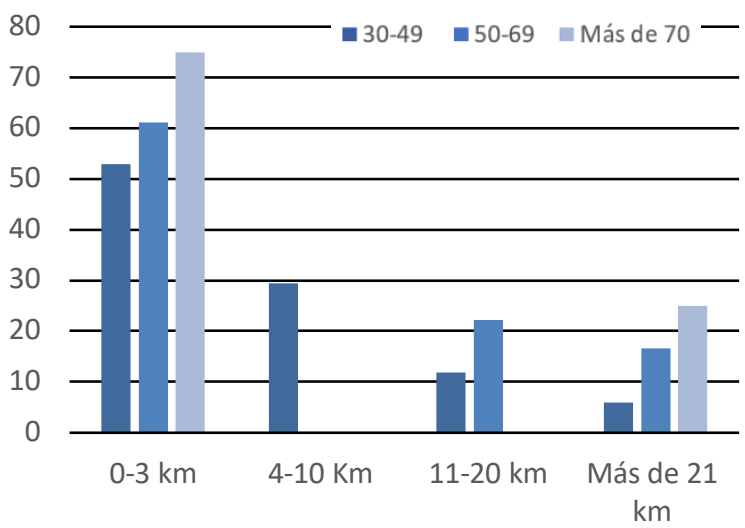

Atendiendo a la mediana, los desaparecidos de entre 30 y 49 años $(m d n=2.96 \mathrm{~km})$, se desplazaron más que los de entre 60 y 69 años ( $m d n=1.69 \mathrm{~km})$, y éstos más a su vez que los de +70 años $(\mathrm{mdn}=0.5 \mathrm{~km})$. Tras realizar las pruebas no paramétricas de la Correlación de Spearman para medir la asociación entre la edad y la distancia, y la Correlación de Kendall para los rangos de edad y la distancia, no se encontraron diferencias estadísticamente significativas $\left(\varrho_{\mathrm{r}}=-0.074 ; \mathrm{p}=0.655 \mathrm{y} \tau_{\mathrm{b}}=-0.080 ; \mathrm{p}=0.627\right.$; respectivamente).

\section{DISCUSIÓN Y CONCLUSIONES}

Analizados los resultados, se ha podido establecer que las personas desaparecidas que cometen suicidio son mayoritariamente varones, y que tienden a utilizar el ahorcamiento como método predilecto. En el caso de las mujeres tienden a escoger la sobredosis como método de suicidio. Estos resultados coinciden con los aportados previamente por la literatura académica (Bergen et al., 2012; Callanan \& Davis, 2011; Yong \& Tzani-Pepelasis, 2019), confirmándose la primera hipótesis (H1).

Se ha podido determinar que los hombres tienden a escoger entornos exteriores, concretamente, prevaleciendo el entorno boscoso. Esto se encuentra relacionado con el método que más frecuentemente utilizan (el ahorcamiento), al tratarse de zonas abundantes en árboles. Las mujeres han tendido escoger entornos situados en el interior, confirmándose así la tercera hipótesis (H3). Asimismo, han sido los hombres los que mayor distancia han recorrido. Estos resultados se asemejan a los obtenidos por la National Crime Agency (2016), confirmándose la quinta hipótesis (H5).

En cuanto a la edad, la distribución de la muestra en los distintos grupos de edad no ha permitido destacar resultados relevantes ni contrastar las hipótesis previas al estudio. La muestra con la que se contaba impedía este hecho, debido a que la gran mayoría se concentraba en los rangos de edad de 30 a 49 años y de 50 a 69 años, y solo se contaba con 4 casos de adultos con más de 70 años. A pesar de ello, se ha podido observar que el ahorcamiento, es el método prevalente para todos los rangos de edad y que los entornos que se encuentran en el exterior son predominantemente escogidos por el grupo de edad de 50 a 69 años.

En lo que respecta al análisis espacial, se observa como la mediana de la distancia desciende conforme la edad aumenta encontrándose que, a mayor edad, menor es la distancia recorrida para llevar a cabo el acto suicida. Sin embargo, las conclusiones alcanzadas no pueden ser comparadas con la literatura consultada puesto que todas las personas desaparecidas de este análisis son mayores de 30 años y todos los estudios analizados hasta la fecha, sobre desplazamiento en desapariciones con respecto a la edad, establecen las diferencias entre menores y mayores de edad.

Cabe destacar la importancia que posee el estudio del método, entorno y desplazamiento para el posterior 
Larrañaga, L., Alfaro, M., García, N. y González, J. L.. (2019). Desapariciones y desenlaces suicidas. Un análisis del método, entorno y desplazamiento. Behavior \& Law Journal, 5(1), 9-17.

desarrollo de herramientas y técnicas de evaluación y respuesta basadas en las líneas llevadas a cabo en esta investigación, así como en combinación con otras futuras. Ello con la intención de mejorar las estrategias de búsqueda ante un caso de desaparición en el que se prevea que existe un riesgo de que la persona desaparecida acabe con su vida. El análisis del método, entorno y desplazamiento más probables según las características del individuo puede facilitar una respuesta rápida y aumentar la posibilidad de encontrar al desaparecido antes de que pueda dañarse a sí mismo, actuando así con carácter preventivo.

$\mathrm{El}$ presente estudio ha generado nuevos interrogantes e ideas que podrán ser utilizadas en futuras líneas, como puede ser la investigación del desplazamiento de los desaparecidos en otro tipo de desapariciones, en pos de comprobar la existencia de diferencias y/o patrones a tener en cuenta.

La principal limitación existente en este estudio ha sido contar con una muestra reducida, lo que ha dificultado el análisis de las variables estudiadas, resultando interesante la replicación de estos análisis con una muestra de mayor tamaño en pos de identificar si existen realmente relaciones y asociaciones estadísticamente significativas. Asimismo, la información recogida a partir de los atestados ha resultado escasa, en ocasiones, para determinar las circunstancias de la muerte, dificultando así un análisis más profundo. Otra de las limitaciones encontradas reside en la carencia de un grupo control de personas que han llevado a cabo el acto de suicidarse sin una denuncia previa por desaparición, que permitiese comparar resultados y observar diferencias y/o patrones entre ambos, como ya han realizado otras investigaciones previas (Woolnough et al., 2019; Sveticic et al., 2012). Ergo, este aspecto queda reservado como una de las principales líneas futuras de investigación.

En definitiva, la carencia de investigaciones centradas en el fenómeno de las desapariciones aboga por la necesidad de fomentar su estudio científico.

\section{REFERENCIAS}

Association of Chief Police Officers (2013). Interim guidance on the management, recording and investigation of missing persons. Londres: College of Policing. Recuperado de: http://library.college.police.uk/docs/college-of-policing/InterimMissing-Persons-Guidance-2013.pdf

Baumeister, R. F. (1990). Suicide as escape from self. Psychological Review, 97(1), 90-113.
Bergen, H., Hawton, K., Waters, K., Ness, J., Cooper, J., Steeg, S. \& Kapur, N. (2012) How do methods of non-fatal self-harm relate to eventual suicide? Journal of Affective Disorders 136, 526-533. DOI: 10.1016/j.jad.2011.10.036.

Biehal, N., Mitchell, F. \& Wade, J. (2003). Lost from view: missing persons in the UK. The Policy Press. Recuperado de: https://www.york.ac.uk/inst/spru/research/pdf/lostFromView. pdf

Bonny, E., Almond, L. \& Woolnough, P. (2016). Adult missing persons: can an investigative framework be generated using behavioural themes? Journal of investigative psychology and offender profiling 13(3), 296-312.

Bowers, L. (2014). Safewards: a model of conflict and containment on psychiatric wards. Journal of Psychiatric and Mental Health Nursing, 21, 499-508. DOI: 10.1002/jip.1459

Camacho-Sandoval, J. (2008). Asociación entre variables: correlación no paramétrica. Acta Médica Costarricense, 50 (3), 144-146. Disponible en: https://www.redalyc.org/articulo.oa?id=43411549004

Callanan, V. \& Davis, M. (2011). Gender differences in suicide methods. Social Psychiatry and Psychiatric Epidemiology 47, 857-869. DOI: $10.1007 / \mathrm{s} 00127-011-0393-5$.

De Leo, D., Conforti, D. \& Carollo, G. (1997). A century of suicide in Italy: a comparison between the old and the young. Suicide and LifeThreatening Behavior 27(3), 239-249. DOI: 10.1111/j.1943278X.1997.tb00406.x.

Domènech, J. (2018). Tratamiento policial de las personas desaparecidas, especial atención a los familiares de los desaparecidos. Centro de Estudios Jurídicos. Recuperado de: https://www.fiscal.es/fiscal/PA_WebApp_SGNTJ_NFIS/desca rga/Ponencia_Domenech_Gustems_Jordi.pdf?idFile=083aaabe2659-4823-acff-d0b6fcdc3dbe

Eales, N. (2017). Risky business? A study exploring the relationship between harm and risk indicators in missing adult incidents. Thesis final. University of Portsmouth. Portsmouth, United Kingdom. Disponible

en: https://researchportal.port.ac.uk/portal/files/11009886/Naomi_ Eales_Thesis_final_copy_Risky_Business.pdf

Foy, S. (2006). Profiling missing persons within New South Wales. Charles Sturt University. Recuperado de: https://missingpersons.gov.au/sites/default/files/PDF\%20\%20Publications/Research/Australian/Profiling\%20Missing\%20 Persons\%20within\%20NSW.pdf

Foy, S. (2016). A profile of missing persons: some key findings for police officers. En: Morewitz S., Sturdy Colls C. (eds) Handbook of Missing Persons. Springer, Cham, 7-18. DOI: 10.1007/978-3-31940199-7_2.

Fyfe, N., Stevenson, O. \& Woolnough, P. (2015). Missing persons: the processes and challenges of police investigation. Policing and Society 25(4), 409-425. DOI: 10.1080/10439463.2014.881812.

García-Barceló, N., Tourís, R.M. \& González, J.L. (2019). Personas desaparecidas: conveniencia de fomentar la investigación científica en España, Boletín Criminológico, artículo 2/2019 ( ${ }^{\circ} 183$ ). Disponible

en www.boletincriminologico.uma.es/boletines/183.pdf

Gibb, J. \& Woolnough, P. (2007). Missing persons: understanding, planning, responding. Recuperado de: https://missingpersons.gov.au/sites/default/files/PDF\%20- 
Larrañaga, L., Alfaro, M., García, N. y González, J. L.. (2019). Desapariciones y desenlaces suicidas. Un análisis del método, entorno y desplazamiento. Behavior Æ Law Journal, 5(1), 9-17.

$\% 20$ Publications/Research/International/Missing $\% 20$ Persons $\% 2$ 0Understanding $\% 20$ Planning $\% 20$ Respondi.pdf

Hawton, K. \& Heeringen, K. (2009). Suicide. Centre for Suicide Research, Lancet. DOI: 10.1016/S0140-6736(09)60372-X.

Hedges, C. (2002). Missing you already: A guide to the investigation of missing persons. London: Home OfficePolice Research Group.

Ministerio del Interior (2018). Informe "Personas desaparecidas" España. Centro Nacional de Desaparecidos. Recuperado de: http://www.lamoncloa.gob.es/serviciosdeprensa/notasprensa/D ocuments/060318INFORME_PERSONASDESAPARECIDAS. pdf

Ministerio del Interior (2019). Informe "Personas desaparecidas" España. Centro Nacional de Desaparecidos. Recuperado de: http://www.interior.gob.es/documents/10180/9814700/2019_0 2_21+INFORME+DE+PERSONAS+DESAPARECIDAS.PD F/72e112d3-ce2f-4653-8527-6e5c5649e4ce

National Crime Agency (2016). iFind. UK Missing Persons Bureau. National Crime Information Center (2017). Missing person and unidentified person statistics. Recuperado de: https://www.fbi.gov/file-repository/2017-ncic-missing-personand-unidentified-person-statistics.pdf/view

Newiss, G. (1999). Missing presumed...? The police response to missing persons. Police Research Series 114. Londres: Home Office. Recuperado http://library.college.police.uk/docs/hopolicers/fprs114.pd

Newiss, G. (2006). Understanding the risk of going missing: estimating the risk of fatal outcomes in cancelled cases. Policing: An International Journal of Police Strategies \& Management 29 (2), 246-260. DOI: 10.1108/13639510610667655.

Newiss, G. (2011). Learning from fatal disappearances: a report by Missing People. Londres: Missing People. Recuperado de: http://www.missingpeople.org.uk/files/Research\%20publication $\mathrm{s} \% 20$ and $\% 20$ presentations/Learning_from_Fatal_Disapearances _2011.pdf

Pedrosa, I., Juarros-Basterretxea, J., Robles-Fernández, A., Basteiro, J. \& García-Cueto, E. (2014). Pruebas de bondad de ajuste en distribuciones simétricas, ¿qué estadístico utilizar? Universitas Psychologica, 14(1), 245-254. DOI: 10.11144/Javeriana.upsy141.pbad

Pértega, S. \& Pita, S. (2004). Asociación de variables cualitativas: el test exacto de Fisher y el test de Mcnemar. Metodología de la Investigación, Fisterra.

Secretaría de Estado de Seguridad (2009). Instrucción 1/2009, de 20 de mayo, sobre la actuación policial ante la desaparición de menores de edad y otras desapariciones de alto riesgo. Secretaría de Estado de Seguridad (2014). Instrucción 14/2014, de 3 de julio, por la que se regula el procedimiento para la activación y funcionamiento del sistema de alerta temprana por desaparición de menores.

Secretaría de Estado de Seguridad (2017). Instrucción 1/2017, de 24 de abril, por la que se actualiza el "protocolo de actuación policial con menores".

Siegel, S. \& Castellan, N.J. (1995). Estadística no paramétrica, aplicada a las ciencias de la conducta. Editorial Trilla: México.

Shalev, K., Schaefer, M. \& Morgan, A. (2009). Investigating missing person cases: how can we learn where they go or how far they travel? International Journal of Police Science \& Management 11(2), 123129. DOI: $10.1350 /$ ijps.2009.11.2.116.

Skogman, K., Alsén, M. \& Öjenhagen, A. (2004). Sex differences in risk factor for suicide after attempted suicide: a follow-up study of 1052 suicide attempters. Social Psychiatry and Psychiatric Epidemiology 39, 113-120. DOI 10.1007/s00127-004-0709-9.

Sveticic, J., San Too, L. \& De Leo, D. (2012). Suicides by persons reported as missing prior to death: a retrospective cohort study. BMJ Open 2, 1-7. Recuperado de: https://www.researchgate.net/publication/221979142_Suicides_ by_persons_reported_as_missing_prior_to_death_A_retrospectiv e_cohort_study.DOI: 10.1136/bmjopen-2011-000607.

Stevenson, E. \& Thomas, S. (2018). A 10 year follow-up study of young people reported missing to the police for the first time in 2005. Journal of Youth Studies, 21 (6), 1-15. DOI:101080/13676261.2018.1268874

Tadros, G., \& Salib, E. (2000). Age and methods of fatal self harm (FSH). Is there a link? International Journal of Geriatric Psychiatry 15, 848-852.

Tang, J., Wu, S., \& Miao, D. (2013). Experimental test of escape theory: accessibility to implicit suicidal mind. Suicide and Life-Threatening Behavior 43(4), 347-355. DOI: 10.1111/sltb.12021.

Tarling, R., \& Burrows, J. (2004). The nature and outcome of going missing: the challenge of developing effective risk assessment procedures. International Journal of Police Science and Management 6(1), $16-26$.

Taylor, C., Woolnough, P., \& Dickens, G. (2018). Adult missing persons: a concept analysis. Psychology. Crime \& Law, 1-24. DOI: 10.1080/1068316X.2018.1529230.

Tinoco, O. (2008). Una aplicación de la prueba chi-cuadrado con SPSS. Revista de la Facultad de Ingeniería Industrial 11(1), 73-77.

Woolnough, P., Magar, E., \& Gibb, G. (2019). Distinguishing suicides of people reported missing from those not reported missing: retrospective Scottish cohort study. BJPsych Open 5(1), 1-6.

Yong, H. \& Tzani-Pepelasis, C. (2019). Suicide and associated vulnerability indicators in adult missing persons: implications for the police risk assessment. Journal of Police and Criminal Psychology. DOI: $10.1007 / \mathrm{s} 11896-018-930$ 